\title{
Evaluation of Why Individuals with ADHD Struggle to Find Effective Digital Time Management Tools
}

\author{
Breanna Desrochers, Ella Tuson, John Magee \\ Clark University, Mathematics and Computer Science Department \\ Worcester, MA 01610, USA \\ \{bdesrochers, btuson, jmagee\}@ clarku.edu
}

\begin{abstract}
After a long history of being viewed as a children's disorder, it is now recognized that Attention-Deficit/Hyperactivity Disorder (ADHD) can affect adults as well. Individuals with ADHD often exhibit work habits significantly different from neurotypical individuals. This paper investigates the use of time management tools among adults with ADHD with the goal of determining where there may be room for improvement in current digital tools so that they may better assist our target demographic. We present findings from a survey of adults with and without ADHD. Our findings indicate dissatisfaction among ADHD adults with the tools currently available to them and highlight key areas for potential development.
\end{abstract}

\section{CCS Concepts}

-Human-centered computing $\rightarrow$ Accessibility systems and tools;

\section{Author Keywords}

Attention-Deficit/Hyperactivity Disorder; ADHD; Time Management; Organization; Survey; Assistive Technology

\section{INTRODUCTION}

Time management is a term colloquially used to refer to a set of skills, often including planning, prioritization, and task activation, relating to the practice of making effective use of one's time for the completion of tasks or goals. It is known that ADHD can impact the development of time management skills and that these skills are important for social functioning and career success [7].

In particular, the Brown Model of ADHD identifies six clusters of functions impaired in individuals with ADHD [4]. One of these clusters, activation, governs skills commonly associated with time management, namely organization, time estimation, prioritizing, and being able to get started on tasks [4]. While there exist many studies on interventions for children and adolescents aimed at teaching organization, time management,

Permission to make digital or hard copies of part or all of this work for personal or classroom use is granted without fee provided that copies are not made or distributed for profit or commercial advantage and that copies bear this notice and the full citation on the first page. Copyrights for thirdparty components of this work must be honored. For all other uses, contact the Owner/Author.

ASSETS '19, October 28-30, 2019, Pittsburgh, PA, USA

C 2019 Copyright is held by the owner/author(s).

ACM ISBN 978-1-4503-6676-2/19/10.

https://doi.org/10.1145/3308561.3354622 and planning skills, few studies examine the types of tools used in these interventions [3, 5, 7].

The National Institute of Mental Health estimates the lifetime prevalence of ADHD among U.S. adults ages 18-44 to be about $8.1 \%$ [2] yet aside from medication, counseling, and cognitive-behavioral therapy, Children and Adults with Attention-Deficit/Hyperactivity Disorder (CHADD) has few recommendations for adults with ADHD on tools and strategies for dealing with the symptoms of their condition [1]. What techniques they do recommend are no different from the general organizational advice one might find on any number of lifestyle blogs written for a much broader audience [1]. In response to this, we set out to determine whether there is a need for organizational and time management tools built with the ADHD community in mind.

The primary research contribution of this paper consists of findings from a survey which featured questions about what time management strategies people use, how effective they are, and where there is room for improvement. The results suggest that young adults with ADHD are generally dissatisfied with the tools for time management that are available to them.

\section{METHODS}

\section{Distribution}

The survey was distributed via Facebook, Reddit, and email. The survey was distributed to both a general campus population as well as to online communities of individuals with ADHD. The survey was hosted on Qualtrics, through which anonymous data was collected.

\section{Participants}

The survey had a total of 55 participants. Respondents self reported being diagnosed with ADHD. Overall, 54.55\% of participants had been diagnosed with ADHD, 40.00\% had not been diagnosed, and $5.45 \%$ were unsure.

The ages of participants ranged from nineteen to forty-seven $(\mathrm{M}=26.91 \mathrm{SD}=7.50)$. Respondents identified as female $(\mathrm{n}=$ $43)$, male $(n=10)$, and non-binary $(n=2)$.

\section{RESULTS \\ Effectiveness of Strategies}

The survey first asked participants to rate how effective their current time management tools are at helping them manage 


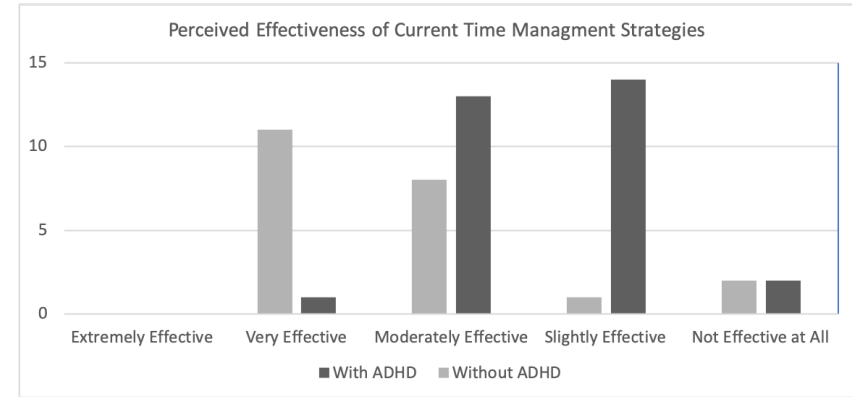

Figure 1. The perceived effectiveness of time management strategies of participants with and without ADHD.

their time. Respondents rated this on a scale from "Extremely Effective" (1) to "Not Effective at All" (5).

No participant reported their tools were "Extremely Effective". Out of all the respondents, $21.82 \%$ rated their tools as "Very Effective", 41.82\% rated as "Moderately Effective", $27.27 \%$ rated as "Slightly Effective", and $9.09 \%$ rated themselves as "Not Effective at All".

\section{Differences Between Those With and Without ADHD}

The survey showed differences in perceived effectiveness based on self reporting an ADHD diagnoses (Fig.1). Using a two tailed t-test, there was a significant difference $(\mathrm{p}<.05)$ between those with ADHD $(\mathrm{M}=3.57 \mathrm{SD}=0.67)$ and those without $\mathrm{ADHD}(\mathrm{M}=2.73 \mathrm{SD}=0.91)$.

\section{Physical versus Digital Tools}

Participants were asked in a multiple choice question if they used exclusively physical tools, exclusively digital tools, or a mixture of both. Physical tools were defined as analog. Digital tools were described as using an application or a website.

Overall, the majority of people used both digital and physical tools when managing their time. Out of all the participants, $67.27 \%$ reported using both physical and digital tools, $23.64 \%$ reported using only physical tools, and $9.09 \%$ reported only using digital tools. The most common digital tools reported were Google Calendar, Apple Calendar, and Reminders.

\section{Differences Between Those With and Without ADHD}

All of the participants who noted using only digital tools also reported being diagnosed with ADHD. The majority of people $(n=8)$ who reported only using physical tools were those without ADHD.

\section{Specific Strategies}

Respondents were also able to report on what specific strategies they used. They did so by choosing from a multiple choice list that incuded an "Other" option that enabled participants to write in if they had another strategy. Participants used to do lists $(n=52)$, calendars $(n=47)$, planners $(n=35)$, timers $(n$ $=18$ ), and more. The majority of participants reported using more than three strategies regularly $(n=40)$.

\section{Differences Between Those With and Without ADHD}

A majority of the types of strategies mentioned previously were used approximately equally by those with ADHD and those without. However, $77.78 \%$ of those who used timers self reported as having ADHD.

\section{DISCUSSION}

\section{Effectiveness of Strategies}

None of the participants rated their current time management strategies as "Extremely Effective", so it is apparent that more work needs to be done in this area in general. Almost $10 \%$ rated their strategies as "Not Effective at All", which implies that they are in need of a tool that can help.

There are a number of potential causes for the difference in perceived effectiveness between those with ADHD and those without ADHD. It has been shown that ADHD can adversely impact the development of time management skills [7], so perhaps these are just natural differences that occur between two groups. This difference could also stem from the fact that many tools are built for neurotypical people, so the technology may not serve the ADHD community as well as it could.

\section{Physical versus Digital Tools}

The majority of people report using both physical and digital tools, with less than a quarter of participants reporting exclusively using physical tools. The responses show a desire to use digital tools, especially among the participants with ADHD. Those with ADHD were the only participants that reported exclusively using digital tools, and very few of those with ADHD exclusively use physical tools.

\section{Specific Strategies}

Overall, the results showed that the majority of the participants practiced multiple different time management strategies. Some tools, physical or digital, may be able to incorporate multiple of these strategies, but it is unclear if participants favor tools that are specialized or are applicable to multiple strategies.

One strategy that was noted as particularity important to the participants with ADHD was the ability to use alarms, alerts, and timers. This may relate to low executive function or hyper focus, both of which are prevalent in those with ADHD and effect one's ability to start or stop tasks $[1,6]$. Alarms, alerts, and timers may be used to mitigate the effects of these states.

\section{CONCLUSION}

The results from this survey show that participants with ADHD find their current time management tools less effective and highlight a need for more tools specifically designed with this population in mind. In particular, there appears to be great room for improvement in the area of digital tools to aid this group, as our results show a preference among individuals with ADHD for the digital tools currently available to them.

Future work for time management strategies for adults with ADHD should focus on confirming the trends suggested by the results of this survey as well as developing new digital tools that incorporate multiple types of time management strategies with a focus on timers.

\section{ACKNOWLEDGMENTS}

NSF support for this project is appreciated (\#IIS-1551590). 


\section{REFERENCES}

1. 2019a. CHADD - Living well with ADHD. (2019). https://chadd.org/

2. 2019b. NIMH » Attention-Deficit/Hyperactivity Disorder (ADHD): The Basics. (2019).

https://www.nimh.nih.gov/health/publications/

attention-deficit-hyperactivity-disorder-adhd-the-basics/ index. shtml

3. Rosanna P. Breaux, Joshua M. Langberg, Stephen J. Molitor, Melissa R. Dvorsky, Elizaveta Bourchtein, Zoe R. Smith, and Cathrin D. Green. 2019. Predictors and Trajectories of Response to the Homework, Organization, and Planning Skills (HOPS) Intervention for Adolescents With ADHD. Behavior Therapy 50, 1 (Jan. 2019), 140-154. D0I :

http://dx.doi.org/10.1016/j.beth.2018.04.001

4. Thomas E. Brown. 2006. Attention Deficit Disorder: The Unfocused Mind in Children and Adults. Yale University Press. Google-Books-ID: ILHWuSekLwUC.
5. Judith Harrison, Bruce Thompson, and Kimberly J. Vannest. 2009. Interpreting the Evidence for Effective Interventions to Increase the Academic Performance of Students With ADHD: Relevance of the Statistical Significance Controversy. Review of Educational Research 79, 2 (June 2009), 740-775. DOI: http://dx.doi.org/10.3102/0034654309331516

6. Soheil Mahdi, Marisa Viljoen, Rafael Massuti, Melissa Selb, Omar Almodayfer, Sunil Karande, Petrus J. de Vries, Luis Rohde, and Sven Bölte. 2017. An international qualitative study of ability and disability in ADHD using the WHO-ICF framework. European Child \& Adolescent Psychiatry 26, 10 (Oct. 2017), 1219-1231. DOI : http://dx.doi .org/10.1007/s00787-017-0983-1

7. Annika Skold. 2007. Self-rating of daily time management in children: psychometric properties of the Time-S: Scandinavian Journal of Occupational Therapy: Vol 24, No 3. Scandinavian Journal of Occupational Therapy 24, 3 (2007). https://www . tandfonline. com/doi/ abs $/ 10.1080 / 11038128.2016 .1185465$ 